\title{
Die Bedeutung von individuellen Merkmalen für die Einschätzung von kollektiven Ressourcen für die Bearbeitung von gemeinsam vereinbarten Zielen in Schulentwicklungsprozessen
}

\section{Zusammenfassung:}

Mit Daten aus dem Schulentwicklungsprojekt RUMBA «Ressourcenentwicklung im Umgang mit Berufsanforderungen» (Vollerhebungen in zwei Schulen) wird aufgezeigt, dass individuelle Merkmale (lehrerbezogene, auf Ressourcenregulation bezogene und kollektive Selbstwirksamkeit) die Einschätzung von kollektiven Qualitätsmerkmalen (wie Teamqualität) mitbestimmen. Im zweiten Teil des Beitrags wird über die statistische Prüfung differenter Entwicklungsverläufe aufgezeigt, dass im Rahmen der evidenzbasierten Schulentwicklungsarbeit gemeinsam vereinbarte Ziele zu angestrebten Veränderungen in den entsprechenden Qualitätsmerkmalen führen. Damit wird deutlich, dass eine gemeinsame Zielfindung eine bedeutende Gelingensbedingung für Schulentwicklungsprozesse darstellt, auch wenn nur eine begrenzte Einigkeit über die Einschätzungen der Ausgangssituation besteht. Entscheidend ist die gemeinsame Relevanzsetzung von anzustrebenden Entwicklungen.

Schlüsselworte: Schulentwicklung, Zielvereinbarung, Selbstwirksamkeit, Teamqualität, Datenfeedback

The Influence of Individual Resources on the Evaluation of Collective Resources as a precondition for Commonly Agreed Goals in School Development and its Effects on School Quality

Based on data out oft the project RUMBA «Ressourcenentwicklung im Umgang mit Berufsanforderungen» (Development of Resources by Dealing with Requirements) for school development, it will be shown, that individual characteristics, such as different kinds of self-efficacy, have an influence on the evaluation of collective resources, such as team quality. In the second part it will be proved, if commonly agreed goals are significant for the changed, caused by the goals. Results show, that goals agreed together, lead to a development, focused on by goal finding processes.

Keywords: School development, goal finding, self-efficacy, team quality, data feedbck

\section{Theoretische Verortung}

Zielvereinbarungen in Schulentwicklungsprozessen sind insbesondere dann erfolgsversprechend, wenn sie von den einzelnen Akteuren mitgetragen werden, deren Überzeugungen den zu verfolgenden Zielen entsprechen und damit zu einem hohen Kommitment in der Bearbeitung derselben beitragen. Da kollektive Ressourcen, wie Teamqualität, Schulqualität und Schulleitungsqualität, individuell verschieden wahrgenommen werden, kann nicht davon ausgegangen werden, dass spezifische Rückmeldungen aus Schulqualitätserhebungen von allen Akteuren gleichermaßen geteilt 
werden. Die Einschätzungen werden individuell verschieden gewichtet, bedingt durch je individuelle Merkmale der Akteure, die als Referenzrahmen die Einschätzungen mitbestimmen. Ausgehend davon, dass individuelle Merkmale die Einschätzung von kollektiven Merkmalen prägen und dass gemeinsam getragene Zielvereinbarungen den Erfolg der Zielerreichung mitbestimmen, stellt sich die Frage nach der Bedeutung von individuellen Ressourcen für die Wahrnehmung von kollektiven Ressourcen. Inwiefern schätzen unterschiedliche Akteure eines Kollegiums kollektive Ressourcen unterschiedlich ein und welche Effekte üben gemeinsam vereinbarte Ziele auf die Entwicklung spezifischer Qualitätsmerkmale aus? Diese Fragen werden im folgenden Beitrag mit Befunden aus zwei Teilstudien des Schulentwicklungsprojekt RUMBA nachgegangen.

Nach einer theoretischen Verortung der zugrundeliegenden Konzepte (Kapitel 1) und der Beschreibung der Projektes RUMBA (Kapitel 2) folgt die Darlegung der Fragestellungen und des methodischen Vorgehens (Kapitel 3). Ergebnisse zur Bedeutung von individuellen Ressourcen, aufgearbeitet an drei Facetten der Selbstwirksamkeit, für die Einschätzung von Merkmalen der Teamqualität (Kapitel 4) werden aufgezeigt, Ergebnisse zu Effekten von gemeinsam vereinbarten Zielen auf die Veränderung von darauf ausgerichteten Qualitätsmerkmalen (Kapitel 5) schließen an und führen in einer Diskussion zum Abschluss des Beitrages (Kapitel 6).

\subsection{Schulqualität und Schulentwicklung}

Wie Schulen eine hohe Schulqualität erreichen ist ein viel diskutiertes Thema. Dabei wird deutlich, dass nicht nur die einzelne Lehrperson gefordert ist, ihr Handeln auf professionelle Qualitätsansprüche auszurichten, sondern auch Schulen als Ganze sollen den Qualitätsansprüchen entsprechen. In den Erwartungen an eine Schule und ihre professionellen Akteure zeichnet sich ein Paradigmenwechsel ab. Von Lehrpersonen wird zunehmend erwartet, ihr berufliches Selbstverständnis weiter zu entwickeln, vom Einzelkämpfer im Sinne von ,Ich und meine Klasse' weg zu kommen und als Teammitglied ein ,Wir und unsere Schule' ins Zentrum der beruflichen Identität zu rücken (z.B. Altrichter/Maag Merki 2010; Fend 2008). Professionelles Handeln ist nicht nur individuell und vor dem Hintergrund der professionellen Standesregeln ( $\mathrm{LCH}$ 2008) zu verantworten, sondern erfolgt eingebettet in eine pädagogische Organisation (Combe/Kolbe 2008) und wird damit von der Kultur der Einzelschule mitgestaltet bzw. durch sie begrenzt (Helsper 2008). Lehrpersonen sind demzufolge nicht nur individuell gefordert, den sich wandelnden Anforderungen zu entsprechen, sondern auch insgesamt im Kollektiv des Kollegiums (Fend 2006). Die individuelle Lehrperson nimmt in ihrem Handlungs- und Wirkungskreis eine bedeutende Rolle im Schulgeschehen ein (Mikroebene) und ist Teil der Qualität der Schule insgesamt (Fend 2006). Akteure der Mikro-Ebene sind Teile des Kollektivs einer Schule und bilden damit eine Einheit auf der Meso-Ebene; Individuen und Kollektiv wirken gegenseitig gestaltend auf einander ein.

Die Qualität von Schulen lässt sich nicht nur über Schülerleistungen beschreiben, sondern auch durch Ausprägungen von Qualitätsmerkmalen des Kollegiums und der Schulleitung, des Schulklimas und des Wohlbefindens der Akteure (Fend 2008; Wenzel 2008). Bestrebungen der Schulentwicklung können demzufolge nicht nur auf verschiedenen Ebenen ansetzen, sondern auch unterschiedliche Aspekte der Schulqualität fokussieren.

Qualitätsmerkmale von Kollegien stellen bedeutende Ressourcen für die Bewältigung beruflicher Anforderungen dar. Durch gesellschaftlich bedingt sich verändernde Erwartungen sind Schulen gefordert, sich Innovationen zu stellen und durch Schulentwicklungsprozesse den Erwartungen zu entsprechen. Gemeinsame Ziele und Werte, ein reflexiver Dialog, 
Zusammenarbeit und eine Bereitschaft, sich auf Veränderungen einzulassen und gemeinsam auf Ziele hin zu arbeiten sind erforderlich, um Schule zu gestalten und den sich verändernden Erwartungen zu entsprechen. Schulen sind somit gefordert, sich mit Gegebenheiten und Erwartungen auseinanderzusetzen und diese entsprechend ihren Ansprüchen und Zielen, sowie entsprechend der an sie gerichteten Erwartungen zu gestalten.

Ressourcentheoretisch (Lazarus/Launier 1998; Buchwald/Schwarzer/Hobfoll 2004) betrachtet bilden kollektive Ressourcen von Kollegien und individuelle Ressourcen der Lehrpersonen Voraussetzungen für die Bewältigung von Berufsanforderungen. Qualitätsmerkmale im Sinne von Ressourcen auf individueller und kollektiver Ebene sind zugleich Voraussetzungen für die Wahrnehmung, Bearbeitung und Bewältigung beruflicher Anforderungen. Dynamisierende Kräfte (wie bspw. Erwartungen, Zielvereinbarungen, Kooperation) führen in Prozesse, welche über neue Erfahrungen die bisherigen Ressourcen verändern (Combe/Gebhard 2009, 550; Keller-Schneider 2010, 115), sowohl auf individueller sowie auf kollektiver Ebene und können auf diese Weise Schulentwicklungsprozesse ermöglichen.

Dem CIPP-Modell von Stufflebeam (1984) folgend lassen sich für Schulentwicklungsprozesse bedeutsame Faktoren in drei Gruppen bündeln, umgeben von Kontextfaktoren, die den Prozess mitbedingen: Input-, Prozess- und Output-Faktoren. Diese lassen sich auf der individuellen sowie auf der kollektiven Ebene erkennen. Individuelle und kollektive Ressourcen stellen als Inputfaktoren Voraussetzungen für Schulentwicklungsprozesse dar, als Outputfaktoren deren Ergebnisse. Für eine Stärkung von Prozessfaktoren, die eine Veränderung anregen, ist von Bedeutung, wie Situationen und daraus hervorgehende Anforderungen wahrgenommen und gedeutet werden, welche Erwartungen daraus hervorgehen, d.h. inwiefern ein Entwicklungsbedarf von den einzelnen Akteuren sowie vom Kollektiv der Akteure wahrgenommen und als relevant gewichtet werden.

\subsection{Wahrnehmung von Anforderungen}

Damit rückt der Prozess der Wahrnehmung, Deutung und Gewichtung von Anforderungen als Voraussetzung für eine in Gangsetzung von Entwicklungen in den Fokus der Aufmerksamkeit. Gemäß stress- und ressourcentheoretischer Zugängen ist erforderlich (Lazarus/Launier 1981; Hobfoll 1989), dass Anforderungen als bedeutend wahrgenommen werden, d.h. den subjektiven Zielsetzungen entsprechen, und dass deren Bewältigung aufgrund der zur Verfügung stehenden Ressourcen als erreichbar betrachtet wird. Als Folge solcher Deutungsprozesse können Erwartungen zu Anforderungen werden und in einen Bearbeitungsprozess münden und neue Erfahrungen ermöglichen. Die daraus hervorgehenden Erkenntnisse gehen in die individuellen Ressourcen ein und bewirken damit eine Veränderung derselben. Aufgrund der veränderten individuellen Ressourcen erscheinen nachfolgende Anforderungen in einem veränderten Referenzrahmen (Keller-Schneider 2010, 113ff.). Diese Transformation spielt sich nicht nur im Kontext von individuellen Deutungsprozessen ab sondern auch in kollektiven (Buchwald/Hobfoll 2004; KellerSchneider/Albisser 2013a).

\section{Individuelle Ressourcen}

Das professionelle Selbst (Bauer/Kopka/Brindt 1996; Bauer 2008) gestaltet im Zusammenwirken unterschiedlicher Komponenten der individuellen Ressourcen die Wahrnehmung und Deutung von Anforderungen mit. Motive (Deci/Ryan 1993), Ziele und 
Zieladaptationen (Heckhausen/Schulz 1995), subjektive Theorien (Gröben/Scheele 2010, 152), Überzeugungen (Blömeke 2008; Reusser et al. 2011; Keller-Schneider 2013), insbesondere Selbstwirksamkeitsüberzeugungen (Schmitz/Schwarzer 2002), wie auch Professionswissen (Shulman 1991; Bromme 1992), tragen zur Einschätzung der Anforderungen bei. Zur Verfügung stehende und für die Bewältigung zu investierende Ressourcen werden vor dem Hintergrund der Ziele und Motive gegeneinander abgewogen (COR-Theorie der Ressourcenerhaltung, Hobfoll 1989). Selbstregulative Fähigkeiten tragen dazu bei, den Prozess des Abwägens von nutzbaren und einzusetzenden Ressourcen wie auch das daraus resultierende Bewältigungshandeln zu steuern (Buchwald/Hobfoll 2004). Je nach Ausgang dieses Abwägungsprozesses werden Anforderungen als Herausforderungen angenommen und bearbeitet - oder sie werden vermieden, zurückgewiesen oder umgedeutet (Keller-Schneider 2010). Eine als nicht relevant oder nicht bewältigbar eingeschätzte Anforderung - sei diese an die individuelle Person gerichtet oder an das Kollektiv einer Schule - wird umgedeutet und negiert. Eine Bearbeitung wird damit vermieden.

Wird eine Anforderung als Herausforderung angenommen und bearbeitet, so führt diese Bearbeitung zu neuen Erfahrungen (Combe/Gebhard 2009, 550), die in die bestehenden Ressourcen integriert, diese verändern und nachfolgende Anforderungen in einen veränderten Referenzrahmen setzen (Keller-Schneider 2010, 115). Für Schulentwicklungsprozesse ist von Bedeutung, dass Anforderungen wahrgenommen und Ausgangsbedingungen als veränderbar eingeschätzt werden, und dass die anzustrebenden Veränderungen als relevant betrachtet werden. Da jedoch individuelle Ressourcen diesen Wahrnehmungs- und Einschätzungsprozess nicht nur auf der individuellen Ebene (KellerSchneider 2010, 229 ff.), sondern auch auf der kollektiven Ebene mitbestimmen (KellerSchneider/Albisser 2012, $170 \mathrm{ff}$. und 2013b, 322 ff.), sind diese für eine Zielvereinbarung in Schulentwicklungsprozessen von Bedeutung.

\section{Kollektive Ressourcen}

Befunde der Schuleffektivitätsforschung weisen darauf hin (z.B. Fend 1998; Scheerens et al. 2003; Bonsen et al. 2008; Bos et al. 2008), dass Merkmale von ,guten' Schulen sowohl mit Merkmalen der Lehrpersonen als auch mit Faktoren der Schule als Ganzes zusammenhängen (Bryk et al. 2010). Ergänzend zu den messbaren Qualitätsmerkmalen (wie Schülerleistungen, Übertritte in weiterführende Schulen) wird die Qualität von Schulen auch von nicht objektiv messbaren Merkmalen wie Klima und Zufriedenheit bestimmt.

Gute Schulen entwickeln und sichern die Lernleistungen ihrer Schüler/innen nicht nur durch die Ausgestaltung von Prozessen auf der Mikro-Ebene - dem Unterricht - sondern auch durch Impulse und Interventionen auf der kollektiven Ebene (Mesoebene), wobei die Schulleitung dabei eine wichtige Funktion einnimmt (vgl. Wissinger 2013). Silins und Mulford (2002) bilanzieren die Schuleffektivitätsforschung mit dem Schluss, dass gegen $20 \%$ der Leistungsvariabilität durch die einzelne Schule selbst bedingt sind. Zu den Faktoren, welche hierfür verantwortlich sein können, zählen Qualitätsmerkmale von Kollegien und Schulleitungen, Schul- und Klassenklima (vgl. Fend 2008). Qualitätsmerkmale von Kollegien ,guter Schulen' (Fend 1998 und 2008; Schweizer/Klieme 2005; Bonsen 2005; Bonsen et al. 2008) umfassen Leistungsorientierung und hohe curriculare Qualität der Lernangebote wie auch spezifische Merkmale von Kollegien und Schulleitungen. Diese betreffen eine hohe Übereinstimmung bezüglich gemeinsamer Ziele und Werte, einen auf das Lernen der Schüler/innen fokussierten reflexiven Dialog, der im gemeinsamen Suchen nach Lösungen auf die Weiterentwicklung von Schul- und Unterrichtsqualität zielt. Konsens und Kohärenz im 
Kollegium, ein lernförderliches Schulklima, wie auch eine auf pädagogische Aufgaben ausgerichtete Schulleitung stellen weitere Merkmale ,guter' Schulen dar. Kooperation gilt als Merkmal ,guter' Schulen und wird dem veränderten Berufsauftrag zufolge von den Lehrpersonen erwartet (vgl. BiD Kanton Zürich).

Ein Kollegium als sozialer Organismus zweiter Ordnung (Bronfenbrenner 1981; Fend 2008) kann als Einheit beschrieben werden, welcher durch die individuellen Lehrpersonen sowie auch vom Kollektiv geprägt wird. Bedingt durch die Gesamtheit der Interaktionen zwischen den Lehrpersonen entwickelt sich eine Eigendynamik, die von Prozessen der Anforderungswahrnehmung des Kollektivs als kollektive Ressourcen mitbestimmt wird und diese gestalten (vgl. Abb. 1).

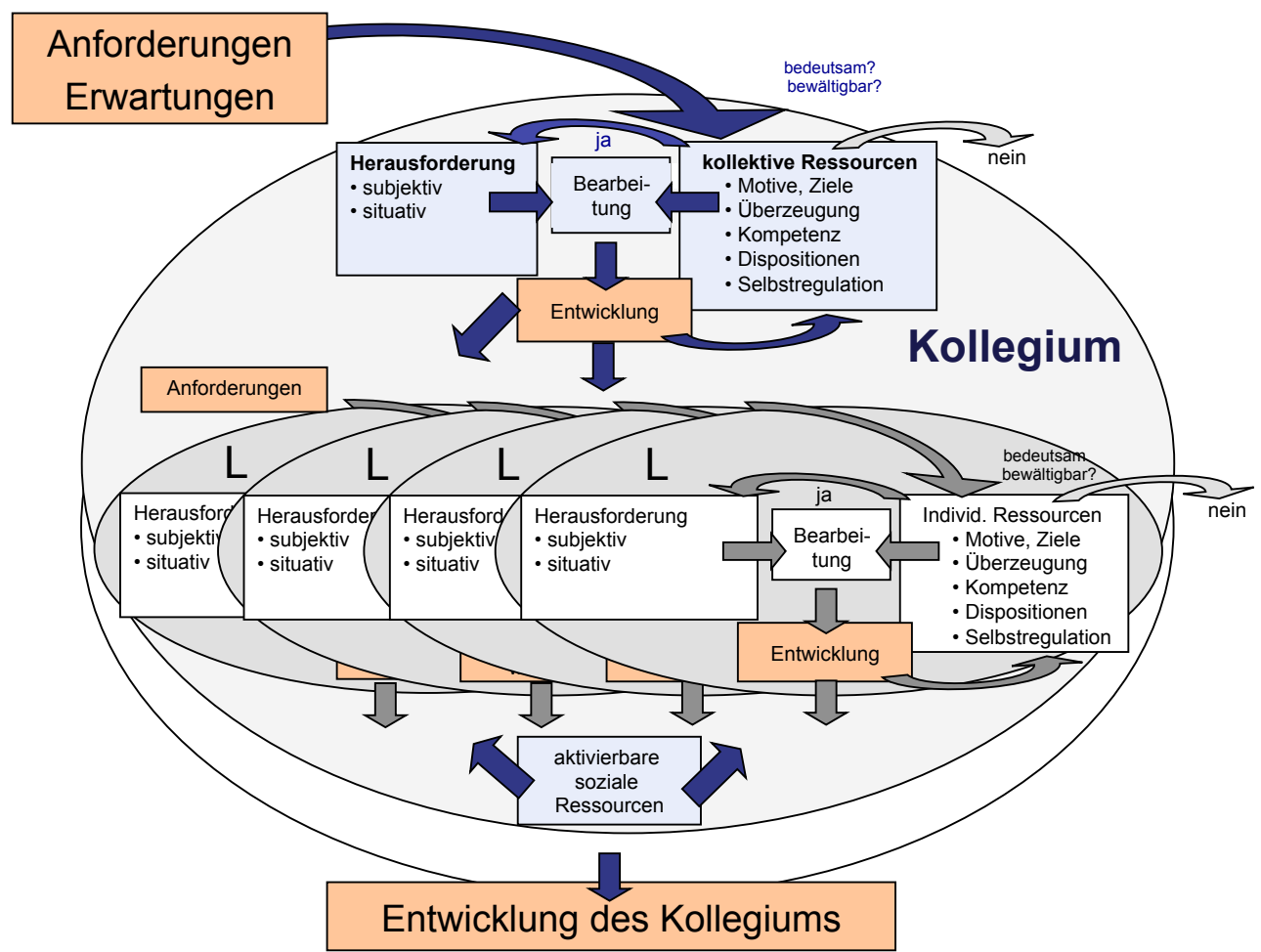

Abb. 1: Prozess der individuellen Wahrnehmung und Bewältigung von Anforderungen im Kontext kollektiver Wahrnehmungsprozesse (vgl. Keller-Schneider/Albisser 2013a, 40).

\subsection{Zielvereinbarungen und ihre Bedeutung für Schulentwicklungsprozesse}

Zielvereinbarungen werden als bedeutsame Steuerungsinstrumente für anzustrebende Veränderungen erkannt. Diese können im Rahmen von Schulentwicklungsprozessen auf der kollektiven Ebene des Kollegiums bzw. der Teams angesetzt werden. Personalentwicklung fokussiert auf Zielvereinbarungen auf der individuellen Ebene der Lehrpersonen, wobei die Schulleitung auf beiden Ebenen die Rolle des Interaktionspartners einnimmt. Zielvereinbarungen und zielführendes Handeln gelten als Faktoren wirksamer Schulen (vgl. Marzano 2003). Nach Rolff (2007) stärken sie den kollektiven Diskurs im Kollegium. Dubs $(2006,113)$ betont, dass gemeinsam verfolgte Ziele und geteilte Werte eine bedeutsame Gelingensbedingung für einen von allen Lehrpersonen mitgetragenen Schulentwicklungsprozess darstellen. Durch die Stärkung von intrinsisch motivierten Zielsetzungen, die ein gemeinsames Bewältigen im Sinne eines ,Communal Coping' (Buchwald 2004, 27ff.) anstreben, wird das Engagement und die Verbindlichkeit erhöht, auf das Ziel hin zu arbeiten. 
Ein sich Involvieren-lassen ist demnach Voraussetzung für die Weiterentwicklung von individuellen aber auch von kollektiven Kompetenzen und Ressourcen. Gemeinsam getragene Zielvereinbarungen fördern Schulentwicklungsprozessen (Dubs 2006). Diesen Prozess in Schulen zu stärken steht im Fokus des Schulentwicklungsprojektes RUMBA (vgl. dazu Keller-Schneider/Albisser 2014).

\section{Das Schulentwicklungsprojekt RUMBA}

Erwartungen allein genügen nicht, um Entwicklungen anzustossen. Ergebnisse von Schulleistungsstudien und Berichten der externen Schulaufsicht setzen Erwartungen an Entwicklungen von Schulen, die von diesen als solche wahrgenommen werden müssen, um an schulentwicklungsrelevanter Bedeutung zu gewinnen (Groß Ophoff et al. 2006). RUMBA ist bestrebt, eine ressourcenorientierte Passung von Zielen in der Schulentwicklungsarbeit zu ermöglichen.

\subsection{Zielsetzungen}

Das Schulentwicklungsprojekt RUMBA «Ressourcenentwicklung im Umgang mit Berufsanforderungen» baut mit der evidenzbasierten und selbstregulierten Team- und Schulentwicklungsarbeit auf stress- und ressourcentheoretischen Zugängen auf. Das Projekt unterstützt Schulen in ihrem Schulentwicklungsprozess. Mittels Datenrückspiegelung zu kollektiven und individuellen Ressourcen sollen Schulen Einblick in die individuellen und kollektiven Ressourcen sowie in das Ausmaß von Übereinstimmungen im Sinne von geteilten Sichtweisen erhalten. Lehrpersonen und Schulleitungen werden mittels Fragebogen zu individuellen Merkmalen, wie Ziele, Motive, Kompetenzen, Überzeugungen und Selbstregulationsfähigkeiten befragt, sowie nach Einschätzungen von Qualitätsmerkmalen auf Schulebene. Um die Schulen in ihrer Weiterentwicklung zu unterstützen und eine Basis für gemeinsam getragene Zielvereinbarungen zu geben - gemäß Dubs $(2006,113)$ für die Nachhaltigkeit der Zielverfolgung bedeutsam - werden den Schulen und den Lehrpersonen Daten zu ihren Einschätzungen der Team-, Schulleitungs- und Schulqualität wie auch zu Ausprägungen ihrer individuellen Merkmale zurückgespiegelt. Die Kollegien erhalten Einblick in die Einschätzungen der Merkmale, über die Streuungen der Einschätzungen (im Rahmen von einer Standardabweichung), die auf Übereinstimmungen und Divergenz der Einschätzungen hinweisen.

Auf diese Weise sollen Schulen darin unterstützt werden, selbstreguliert nach eigenen Relevanzsetzungen Zielvereinbarungen zu treffen, die aus der Sicht der Akteur/innen bedeutsame und erreichbare Ziele fokussieren, und auf die es sich aus der subjektiven aber geteilten Sichtweise des Kollegiums lohnt hinzuarbeiten. Durch die Nutzung von eigenen Schwerpunktsetzungen als Ausgangspunkt für die Schulentwicklungsarbeit beabsichtigt das Vorgehen eine optimale Passung der vereinbarten Ziele zu erreichen und damit eine starke Einbindung der Akteur/innen in gemeinsam getragene Entwicklungen zu ermöglichen.

\subsection{Der Prozess der Datenerhebung und Datennutzung}

Die Datenerhebung im Prä-Post-Design mittels Fragebogen rahmt eine Intervention, die aus der Datenrückspiegelung durch die Projektleitung und der Datennutzung durch die Lehrpersonen und die Schulleitungen besteht (vgl. Abb. 2).

Datenerhebung: Mittels Fragebogen werden Lehrpersonen und die Schulleitung ganzer Schulen (Vollerhebungen) nach Einschätzungen der Schul-, Team- und Schulleitungsqualität befragt, sowie auch nach individuellen Merkmalen (Berufsmotive, Überzeugungen, 
Selbstregulationsfähigkeiten, Kompetenzerleben, Veränderungsbereitschaft und Kooperationsverhalten).

Datenrückspiegelung: Die Rückspiegelung von Ergebnissen zu individuellen und kollektiven Ressourcen soll den Schulen als Ausgangspunkt für evidenzbasierte Schul- und Personalentwicklungsprozesse dienen (vgl. Gathen, 2006, 79; Peek 2006, 1345 ff.; Schneewind 2006, 119 f.).

Im Rahmen von regulären Teamsitzungen werden den Kollegien und der Schulleitung Ergebnisse zu kollegiumsbezogenen Einschätzungen vorgestellt. Über den Einblick in Mittelwerte wird der durchschnittliche Wert der Einschätzungen verdeutlicht, über Streuungen der Grad der Einigkeit bzw. der Uneinigkeit der Einschätzungen. Aus Übereinstimmungen und Divergenzen wie auch aufgrund von Ausprägungen einzelner Qualitätsmerkmale werden Schulen angeregt, entsprechend ihren Relevanzsetzungen evidenzbasiert Ziele zu vereinbaren, die im Zuge der weiteren Schulentwicklungsarbeit in den Fokus gerückt werden. Das Vorgehen spricht den Mitgliedern des Kollegiums und der Schulleitung einen Gestaltungsfreiraum zu, so dass Schulentwicklungsprozesse ressourcenbezogen und ressourcenerhaltend erfolgen können. Die gemeinsame Verständigung geschieht über Ergebnisrückmeldungen der Einschätzungen des Kollektivs.

Datennutzung: Der Prozess der Datennutzung ist dem Modell von Hosenfeld und Groß Ophoff $(2007,358)$ folgend in einzelne Stationen gegliedert (vgl. Abb. 2). Im Rahmen des Projekts wurden in Zusammenarbeit mit den ersten Projektschulen Instrumente entwickelt, die im Zuge des Datennutzungsprozesses von den Schulleitungen und den Teams eingesetzt werden können (vgl. Keller-Schneider/Albisser 2014).

In einer Informationsveranstaltung (Station 1) erhalten Lehrpersonen und Schulleitung ausgewählte Ergebnisse der ersten Datenerhebung (schriftliche Befragung t1) zurück. Eine individuelle Rezeption (Station 2) bildet die Ausgangslage für Zielvereinbarungen im Team (Station 3). In einer danach anschließenden Umsetzung und in halbjährlichen Zwischeneinschätzungen (Station 4) wird die Schulentwicklungsarbeit reflektiert. Eine Abschlusseinschätzung der Zielerreichung (5) schließt den Bogen.

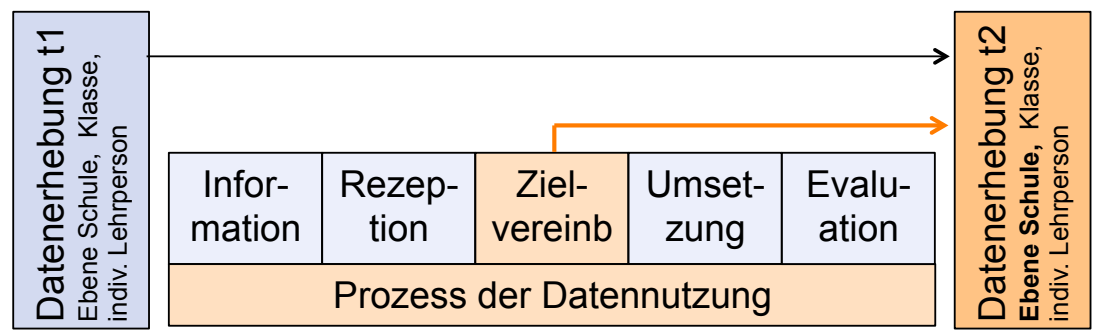

Abb. 2: Stationen des Datennutzungsprozesses, umfasst von fragebogengestützten Datenerhebungen im PräPost-Design.

Zweite Datenerhebung nach Abschluss der Intervention: Ergebnisse einer zweiten Datenerhebung mittels Fragebogen im Rahmen von Teamarbeitszeit (Vollerhebung) ermöglicht, Veränderungen der kollektiven und individuellen Ressourceneinschätzungen zu erkennen (Befragung t2). 


\section{Fragestellungen und methodisches Vorgehen}

\subsection{Fragestellungen}

Wenn davon ausgegangen werden kann, dass individuelle Ressourcen für die Wahrnehmung und Deutung von Anforderungen, die als Erwartungen an die Lehrpersonen individuell sowie an Kollegien insgesamt gerichtet werden, von Bedeutung sind (KellerSchneider 2010), dass diese auch die Einschätzung von kollektiven Ressourcen mitbestimmen (Keller-Schneider/Albisser 2011 und 2012a) und dass eine darauf basierende Zielverfolgung gemeinsam getragen werden soll (Dubs 2006), um als Prozessfaktoren zur Optimierung von Schulqualität beizutragen (Stufflebeam 1984), dann stellen sich folgende Fragen:

1) Welche Bedeutung üben individuelle Ressourcen, operationalisiert über Aspekte der Selbstwirksamkeit, auf die Einschätzung von kollektiven Ressourcen der Teamqualität aus?

2) Welche Effekte gehen von gemeinsam vereinbarten Zielen auf die Veränderung der entsprechenden Merkmale der Teamqualität aus?

\subsection{Design und Stichprobe}

Zur Prüfung dieser Fragestellungen werden Daten aus dem Schulentwicklungsprojekt RUMBA genutzt (1. Phase 2010-2012). Im Pre-Post Design, welche eine Intervention umfassen, werden mittels Fragbogen Daten zu individuellen und kollektiven Ressourcen erhoben. Ergebnisse werden den Schulen zurückgespiegelt und als Grundlage der evidenzbasierten, gemeinsam getragenen Schulentwicklungsarbeit genutzt. Die Intervention besteht darin, dass die Schulen nach individuellen Gewichtungen der erhaltenen Ergebnisse im gemeinsamen Diskurs gemeinsam getragene Schwerpunkte für die weitere Schulentwicklungsarbeit setzen und diese in Zielvereinbarungen festlegen. Mittels in den Teams bearbeiteten Fragebogen, die im Rahmen der Intervention als Reflexionsinstrument eingesetzt wurden, schätzen die Teams gemeinsam die aktuelle Situation ein, um den Anfangspunkt einer möglichen Entwicklung festzuhalten. Nach rund eineinhalb Jahren erfolgt eine Einschätzung der Zielerreichung sowie eine zweite Fragebogen Erhebung (vgl. Abb. 2).

Die Stichprobe umfasst 93 Lehrpersonen von zwei Primarschulen (Vollerhebung) im Kanton Zürich. Die Kollegien der beiden Schulen unterscheiden sich weder bezüglich Geschlecht (40 bzw. 38 Frauen zu 6 bzw. 5 Männer), Berufserfahrung ( $M=16.8$ bzw. 15.8, $\mathrm{SD}=11$ bzw. 9.9 Jahre), Funktionen (je 60\% sind in Aufgaben der Klassenlehrperson involviert), noch Beschäftigungsgraden $\left(\mathrm{Chi}^{2}(4, \mathrm{n}=88)=4.134, \mathrm{p}=.39\right)$.

\subsection{Instrumente:}

In der Fragebogenerhebung des Projekts RUMBA wurden zahlreiche Instrumente eingesetzt (vgl. dazu Keller-Schneider/Albisser 2014), für die Ergebnisse dieses Beitrags sind folgende von Bedeutung:

\section{Individuelle Merkmale der Lehrpersonen}

Individuelle Ressourcen werden in dieser Publikation über drei unterschiedliche Zugänge der Selbstwirksamkeit operationalisiert. Dazu wurde die Skala der lehrerbezogenen Selbstwirksamkeit von Schwarzer und Jerusalem (1999) eingesetzt (Beispielitem: ,Ich weiss, dass ich es schaffe, selbst den problematischsten Schüler/innen den Stoff zu vermitteln.'), sowie die Skala zur kollektiven Selbstwirksamkeit von Schwarzer und Jerusalem (1999), als individuelle Sicht auf die kollektiven Ressourcen (Bsp.: ,Auch mit außergewöhnlichen 
Vorfällen können wir zurechtkommen, da wir uns im Kollegium gegenseitig Rückhalt bieten.'). Als dritter Zugang wird die Skala der im Rahmen von RUMBA entwickelten ressourcenbezogenen Selbstwirksamkeit genutzt, als Überzeugung, die eigenen Kräfte und den Ressourceneinsatz regulieren zu können (Entwicklung ausgehend von Items zur KellerSchneider 2010, vgl. Tabelle 1).

Tab. 1: Items zur Skala der auf Ressourcenregulation bezogenen Selbstwirksamkeit

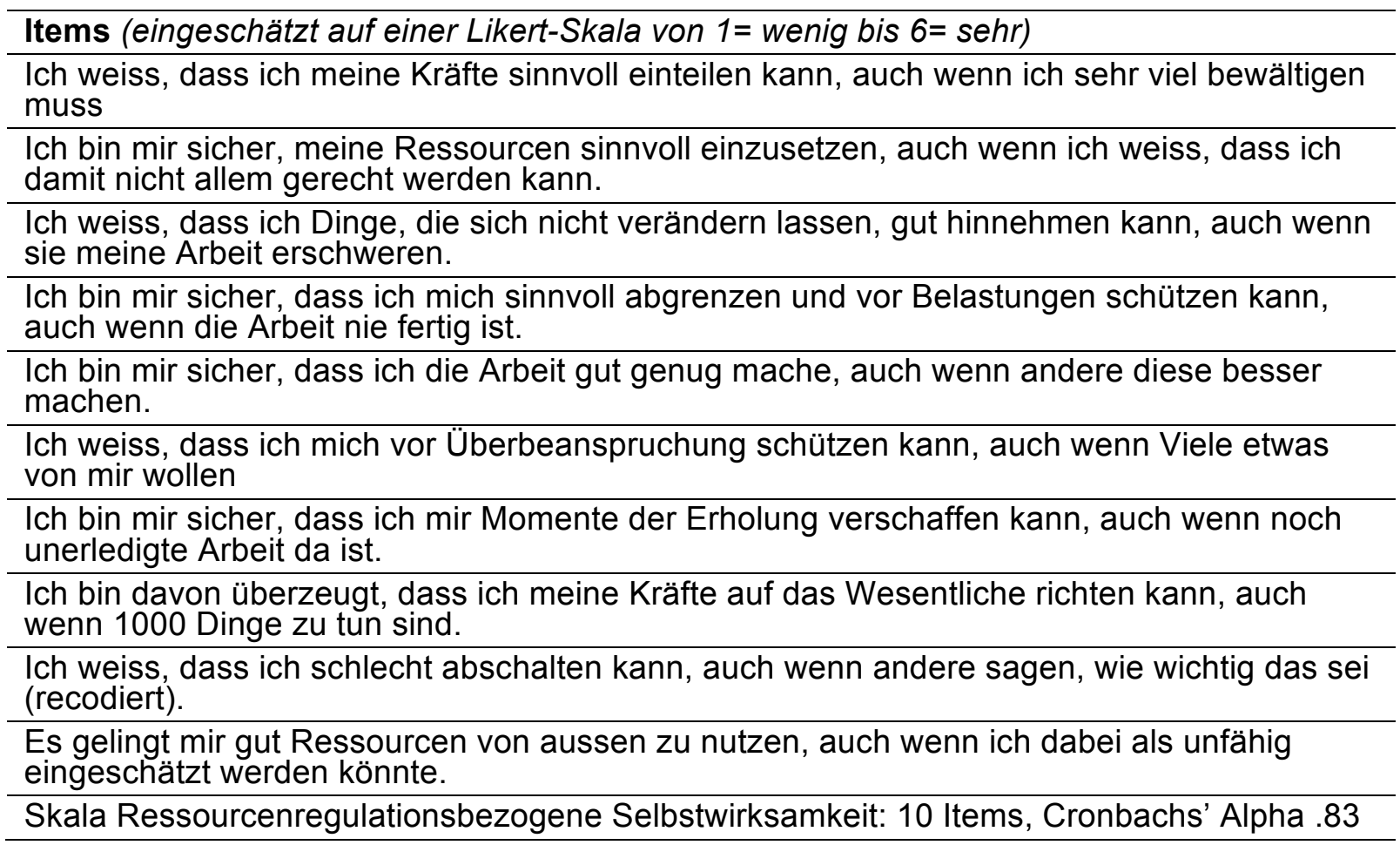

\section{Kollektive Ressourcen des Kollegiums}

Diese werden mittels des im Rahmen des Projekts RUMBA entwickelten Instrumentariums (vgl. Keller-Schneider/Albisser 2014) erhoben. Für die Beantwortung der ersten Fragen werden kollektive Ressourcen über Aspekte der Teamqualität operationalisiert (vgl. dazu Keller-Schneider/Albisser 2013a). Ausgehend von empirisch erhobenen Qualitätsmerkmalen von Kollegien (Fend 1998; Scheerens et al. 2003; Bonsen et al. 2008) wurde auf der Basis bestehender Instrumente, mit auf Schweizer Verhältnisse angepassten Neuentwicklungen ergänzt, ein Instrument mit faktoranalytisch gebildeten Skalen entwickelt. Aus diesem Instrument werden für die Beantwortung der Frage 1 sechs Skalen herausgegriffen, die in der Schulentwicklungsforschung bedeutende Qualitätsmerkmale erfassen.

Für die Beantwortung der in Fragstellung 2 zu prüfenden Veränderungen von kollektiven Ressourcen werden mit den folgenden Instrumenten erhobene Daten genutzt: 1) Schulkultur (vgl. Schwarzer/Jerusalem 1999), Unterrichtsbezogene Kooperation (Keller-Schneider/ Albisser 2013a), Resignationstendenz (aus Schaarschmidt/Fischer 1996).

\subsection{Auswertung}

Zur Fragestellung 1: Um Effekte von Aspekten der Selbstwirksamkeit auf die Einschätzung von Merkmalen der Teamqualität zu prüfen, wurden über die Ausprägungen der lehrerbezogenen, ressourcenregulationsbezogenen und kollektiven Selbstwirksamkeit mittels einer Clusteranalyse (Methode Ward, quadrierte euklidische Distanz) Typen gebildet, die 
varianzanalytisch nach Unterschieden in den Einschätzungen ausgewählter Merkmale der Teamqualität geprüft werden.

Zur Fragestellung 2: Zur Prüfung der Effekte der Zielvereinbarung auf die Ausprägungen der darauf bezogenen Qualitätsmerkmale der kollektiven Ressourcen wurde im Kontrollgruppendesign varianzanalytisch geprüft, inwiefern sich die jeweiligen Interventionsgruppen am Ende der, von den jeweiligen Kontrollgruppen unterscheiden.

\section{Ergebnisse}

\subsection{Zusammenwirken von individuellen und kollektiven Ressourcen - Teamqualität aus der Sicht von Lehrpersonen unterschiedlicher Profile der Selbstwirksamkeit}

Typen unterschiedlicher Profile über Facetten der Selbstwirksamkeit

Abbildung 3 zeigt die Ausprägungen der erhobenen Teilaspekte der Selbstwirksamkeit in zWerten, wodurch die Abweichungen vom Mittelwert der Stichprobe insgesamt verdeutlicht werden.

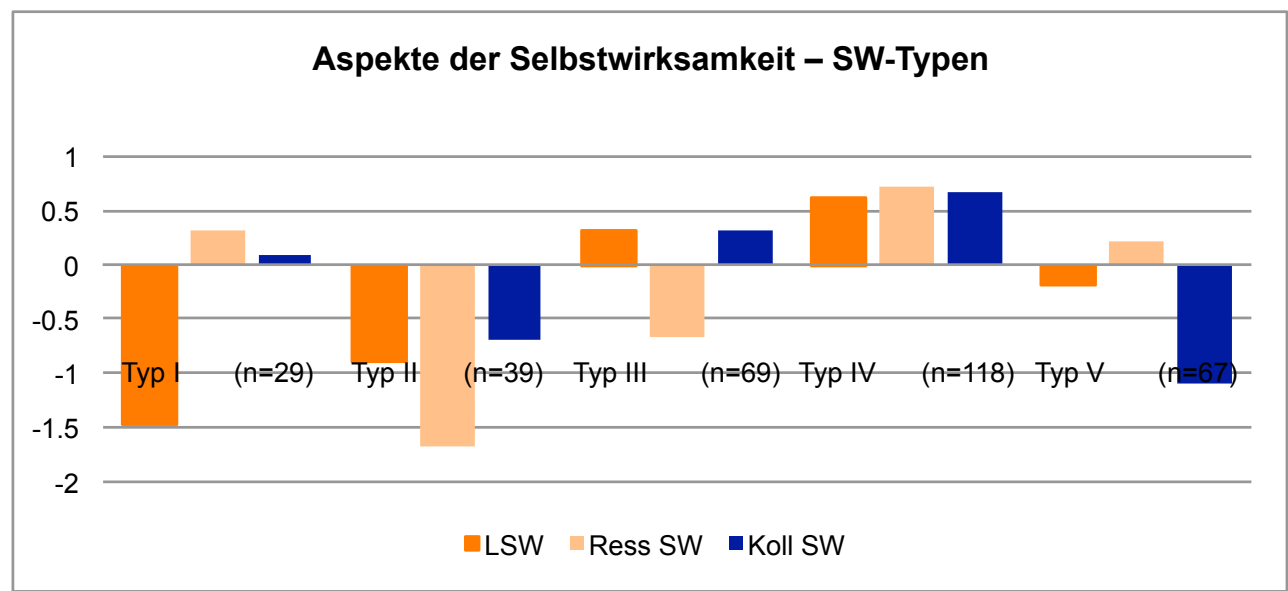

Abb. 3: Typen mit unterschiedlichen Profilen in den Ausprägungen von Facetten der Selbstwirksamkeit (Clusteranalytisch gebildete Typen und z-Werte dargestellt)

Drei Typen (I, III, V) zeichnen sich je spezifisch durch einen unterdurchschnittlich ausgeprägten Aspekt der Selbstwirksamkeit aus, bei durchschnittlicher Ausprägung der beiden anderen. Bei Typ I ist das die lehrerbezogene Selbstwirksamkeit, bei Typ III die ressourcenregulationsbezogene und bei Typ V die kollektive. Zwei Typen weisen ausgewogene Profile auf, wobei Typ II in allen drei Aspekten der Selbstwirksamkeit unterdurchschnittliche Werte aufweist, Typ IV in allen drei Bereichen überdurchschnittlich ausgeprägte Werte. Diese charakteristischen Profile zeugen zudem von einer Unabhängigkeit dieser drei Facetten der Selbstwirksamkeit. Unterschiedliche Facetten von Selbstwirksamkeit sind differentialdiagnostisch von Bedeutung.

\section{Typenspezifische Einschätzungen von Aspekten der Teamqualität}

Ergebnisse in Abbildung 4 und in Tabelle 2 zeigen auf, inwiefern und in welchem Ausmaß sich die Typen in der Einschätzung von Teamqualitätsmerkmalen unterscheiden. Die Einschätzungen verlaufen weitgehend parallel, was darauf hinweist, dass sich die Selbstwirksamkeitsprofile homolog auf die Einschätzung unterschiedlicher Teamqualitätsmerkmale auswirken. Individuelle Ressourcen, hier erfasst über Selbstwirksamkeitsüberzeugungen prägen als Filter die Wahrnehmung von kollektiven Ressourcen mit. 
Die Typen II und V, die beide unterdurchschnittliche Werte in der Einschätzung der kollektiven Selbstwirksamkeit aufweisen, zeigen die tiefsten Werte in den Ausprägungen der der Teamqualität, die als kollektive Ressource zur Bewältigung der beruflichen Anforderungen beitragen. Typ IV mit überdurchschnittlichen Ausprägungen in allen drei erfassten Selbstwirksamkeitsorientierungen zeichnet sich durch die höchsten Werte der Teamqualitätsmerkmale aus. Die Werte der Typen III und I liegen dazwischen.

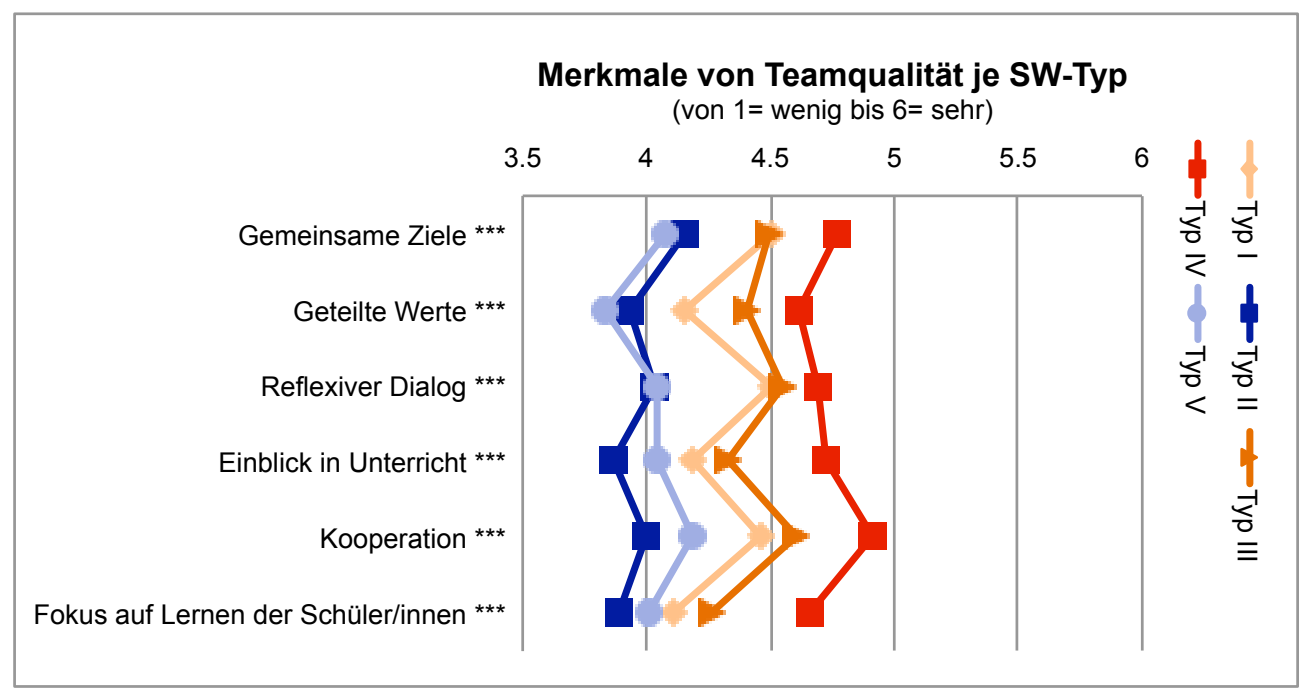

Abb. 4: Einschätzungen von Qualitätsmerkmalen der Teamqualität je Typ unterschiedlicher Profile über Facetten der Selbstwirksamkeit

Tab. 2: Mittelwerte der eingeschätzten Merkmale der Teamqualität je Typ mit Ergebnissen der multifaktoriellen Varianzanalysen (GLM) und der Post Hoc Tests

\begin{tabular}{|c|c|c|c|c|c|c|c|c|}
\hline $\begin{array}{l}\text { Merkmale der } \\
\text { Teamqualität }\end{array}$ & Typ I & Typ II & Typ III & Typ IV & Typ V & Total & GLM & Post Hoc \\
\hline Gemeinsame Ziele & 4.50 & 4.16 & 4.49 & 4.77 & 4.08 & 4.47 & $\star * *$ & $V<|I|<|I|<\mid<<^{*} I V$ \\
\hline Geteilte Werte & 4.16 & 3.93 & 4.40 & 4.61 & 3.83 & 4.28 & $* * *$ & $\mathrm{~V}<1 \mathrm{l}<\mathrm{I}<\mathrm{II}<\mathrm{IV}$ \\
\hline Reflexiver Dialog & 4.50 & 4.03 & 4.55 & 4.69 & 4.04 & 4.43 & $* * *$ & ||$<V<{ }^{*}|<| I \mid<I V$ \\
\hline Einblick in Unter. & 4.18 & 3.86 & 4.33 & 4.73 & 4.04 & 4.35 & $\star \star *$ & $I I<\mathrm{V}<\mathrm{l}<\mathrm{III}<<^{* *} \mathrm{IV}$ \\
\hline Kooperation & 4.46 & 4.00 & 4.60 & 4.91 & 4.18 & 4.54 & $\star \star * *$ & $I I<V<1<1 \| \mid<^{*} I V$ \\
\hline Fokus auf Lernen & 4.11 & 3.88 & 4.26 & 4.66 & 4.01 & 4.30 & $* * *$ & $I|<V<1<I I|<<^{* *} \mid V$ \\
\hline
\end{tabular}

\subsection{Zielvereinbarungen und ihre Bedeutung für die Qualitätsentwicklung}

Dargestellt werden drei Zielvereinbarungen, die von mehreren Teams vereinbart wurden. An je einem Beispiel wird exemplarisch eine mittels des dafür entwickelten Instruments vorgenommene Zieleinschätzung zu Beginn sowie beim Abschluss der Intervention dargestellt (vgl. Tab. 3 bis 5). Ausgewählt wurden Zielvereinbarungen, die von mehreren Teams fokussiert wurden. Teams, welche an der je spezifischen Zielvereinbarung arbeiteten, werden zur Interventionsgruppe zusammengefasst und diejenigen, die nicht an dieser Zielvereinbarung arbeiteten, zur Kontrollgruppe. Mittels Varianzanalysen wurden Unterschiede zwischen Interventions- und Kontrollgruppen geprüft, entsprechende Ergebnisse sind in Tabelle 6 zusammengefasst. 


\section{Zielvereinbarung 1: Einheitliche Handhabung der Pausenaufsicht}

Sechs von neun Teams setzen sich zum Ziel, die Aufgaben der Pausenaufsicht zu klären und einheitlicher zu handhaben. Die relativ tiefen Werte der diesbezüglichen Einschätzungen und ihre Bedeutsamkeit in der individuellen und kollektiven Gewichtung der Teammitglieder bilden den Ausgangspunkt für die Optimierungsabsichten.

Tab. 3: Reflexion der Leitfragen bei Zielvereinbarung und Zwischeneinschätzung der einheitlichen Handhabung der Pausenaufsicht

\begin{tabular}{|c|c|c|}
\hline Leitfrage: & Zielvereinbarung & Zwischeneinschätzung \\
\hline 1) Wo stehen wir jetzt? & $\begin{array}{l}\text { 4 } \\
(1=\text { am Anfang, 10= am Ziel) }\end{array}$ & $\begin{array}{l}8 \\
(1=a m \text { Anfang, 10= am Ziel) }\end{array}$ \\
\hline $\begin{array}{l}\text { 2a) Woran erkennen wir } \\
\text { das? }\end{array}$ & $\begin{array}{l}\text { Gemäß Rückmeldungen der } \\
\text { Schüler/innen ist unklar, was } \\
\text { gilt }\end{array}$ & $\begin{array}{l}\text { Diskussionen in den Pausen } \\
\text { mit Kindern, welche die } \\
\text { Pausennlatzregeln nicht }\end{array}$ \\
\hline $\begin{array}{l}\text { Was ist bereits } \\
\text { vorhanden? }\end{array}$ & $\begin{array}{l}\text { Pausenregeln, } \\
\text { Schulhausregeln, kurzer } \\
\text { Austausch in Pausen, } \\
\text { Absprachen situativ }\end{array}$ & $\begin{array}{l}\text { befolgen, sind seltener. } \\
\text { Die Kinder klären Probleme } \\
\text { häufig selber. }\end{array}$ \\
\hline $\begin{array}{l}\text { 2b) Was hat sich } \\
\text { verändert? }\end{array}$ & Zwischenevaluationsfrage & $\begin{array}{l}\text { Das erwartete Verhalten ist den } \\
\text { Kindern klarer und bewusster. }\end{array}$ \\
\hline $\begin{array}{l}\text { 2c) Was haben wir aktiv } \\
\text { beigetragen, damit } \\
\text { sich etwas verändern } \\
\text { konnte? }\end{array}$ & Zwischenevaluationsfrage & $\begin{array}{l}\text { Pausenregeln reduziert, im } \\
\text { Klassen- und Schülerrat sowie } \\
\text { in Teamsitzungen geklärt. }\end{array}$ \\
\hline $\begin{array}{l}\text { 3) Was können wir aktiv } \\
\text { beitragen, um } \\
\text { weiterzukommen } \\
\text { weiter zu kommen? }\end{array}$ & $\begin{array}{l}\text { Vorschläge einbringen, } \\
\text { verbindliche Lösungen } \\
\text { vereinbaren und sich daran } \\
\text { halten. }\end{array}$ & $\begin{array}{l}\text { Pausenregeln regelmäßig } \\
\text { verdeutlichen und bei } \\
\text { Missachtung direkt } \\
\text { intervenieren. }\end{array}$ \\
\hline $\begin{array}{l}\text { 4) Was kann die } \\
\text { Schulleitung dazu } \\
\text { beitragen? }\end{array}$ & $\begin{array}{l}\text { Zeitfenster in den } \\
\text { Teamsitzungen gewähren; } \\
\text { Diskussionszeit mit dem } \\
\text { Schüler/innenrat } \\
\text { koordinieren. }\end{array}$ & $\begin{array}{l}\text { Die Pausenregeln am Anfang } \\
\text { des Schuljahres in einer } \\
\text { Teamsitzung klären. }\end{array}$ \\
\hline $\begin{array}{l}\text { 5) Wie groß ist unsere } \\
\text { Hoffnung, dass sich } \\
\text { etwas ändert? }\end{array}$ & $\begin{array}{l}9 \\
(1=\text { gering bis } 10=\text { gro } \beta)\end{array}$ & $\begin{array}{l}8 \text { Wir sind deutlich weiter }(:) . \\
(1=\text { gering bis } 10=\text { groß })\end{array}$ \\
\hline
\end{tabular}

Aus diesen im Team erhobenen Einschätzungen geht hervor, dass die Einschätzungen der aktuellen Situation (Wo stehen wir jetzt?) einen deutlichen Anstieg aufweisen (vom Skalenpunkt 4 auf 8). Es kann angenommen werden, dass eine Fokussierung auf diese Zielsetzung, die durch konkrete Vorgehensweisen angegangen und deren Beachtung bzw. Missachtung im Kollegium diskutiert werden kann, zu einer Entwicklung in die gewünschte Richtung und zu einer Qualitätssteigerung beigetragen hat.

Werden die Veränderungen über eine varianzanalytische Prüfung der fragebogenbasierten Einschätzungen zu Beginn und am Ende der Intervention geprüft, so zeigt sich auch da ein statistisch bedeutsamer Anstieg der Ausprägungen bei mittleren Effektstärken (vgl. Tabelle 6). In den Einschätzungen besteht darüber hinaus eine größere Übereinstimmung, was an den geringeren Streuungen erkennbar ist. In der Kontrollgruppe zeigen sich keine statistisch bedeutsamen Veränderungen.

\section{Zielvereinbarung 2: Unterrichtsbezogene Kooperation stärken}

Aufgrund der relativ gering eingeschätzten Ausprägungen bei hoher Bedeutsamkeit des Qualitätsmerkmals haben sich vier von elf Teams auf eine darauf bezogene Zielsetzung geeinigt. In Tabelle 4 werden die entsprechenden Einschätzungen aufgeführt, Tabelle 6 zeigt 
die Ergebnisse der statistischen Prüfung der Entwicklungen, erfasst über die Fragebogenerhebungen vor und nach der Intervention. In der Interventionsgruppe ist ein statistisch sehr bedeutsamer Anstieg bei hoher Effektstärke bei gleich bleibenden Streuungen zu verzeichnen.

Tabelle 4: Reflexion der Leitfragen bei Zielvereinbarung und Zwischeneinschätzung der Stärkung unterrichtsbezogener Kooperation

\begin{tabular}{|c|c|c|}
\hline Leitfrage: & Zielvereinbarung & Zwischeneinschätzung \\
\hline 1) Wo stehen wir jetzt? & $\begin{array}{l}5 \\
(1=\text { Anfang, 10= am Ziel) }\end{array}$ & $\begin{array}{l}5 \\
(1=\text { Anfang, } 10=\text { am Ziel) }\end{array}$ \\
\hline $\begin{array}{l}\text { 2) Woran erkennen wir, } \\
\text { dass das Team an } \\
\text { diesem Punkt steht? } \\
\text { Was ist vorhanden? }\end{array}$ & $\begin{array}{l}\text { Materialaustausch; } \\
\text { offene ungeplante auf den } \\
\text { Unterricht bezogene } \\
\text { Gespräche. }\end{array}$ & $\begin{array}{l}\text { Materialaustausch und } \\
\text { Gespräche finden in 2er- } \\
\text { Gruppen statt; Zimmertüren } \\
\text { sind häufig offen. }\end{array}$ \\
\hline $\begin{array}{l}\text { 2b) Was hat sich } \\
\text { verändert? }\end{array}$ & Zwischenevaluationsfrage & $\begin{array}{l}\text { Durch die neu dazu } \\
\text { gekommenen Lehrpersonen } \\
\text { ändert sich die } \\
\text { Zusammenarbeit. }\end{array}$ \\
\hline $\begin{array}{l}\text { 2c) Was haben wir aktiv } \\
\text { beigetragen, damit sich } \\
\text { etwas verändern } \\
\text { konnte? }\end{array}$ & Zwischenevaluationsfrage & $\begin{array}{l}\text { Noch nicht viel. Wir } \\
\text { strukturieren die } \\
\text { pädagogischen Teams um, } \\
\text { um besser arbeiten zu } \\
\text { können. }\end{array}$ \\
\hline $\begin{array}{l}\text { 3) Was können wir aktiv } \\
\text { beitragen, um weiter zu } \\
\text { kommen? }\end{array}$ & $\begin{array}{l}\text { Kopierraum zum Vorbereiten } \\
\text { und Korrigieren nutzen; offen } \\
\text { legen, wer in den Ferien wann } \\
\text { in der Schule arbeitet. }\end{array}$ & $\begin{array}{l}\text { Liste mit } \\
\text { Unterrichtsmaterialien } \\
\text { aktualisieren, Sitzungszeiten } \\
\text { im Pädagogischen Team zur } \\
\text { Planung von Unterricht } \\
\text { nutzen. }\end{array}$ \\
\hline $\begin{array}{l}\text { 4) Was kann die } \\
\text { Schulleitung dazu } \\
\text { beitragen? }\end{array}$ & $\begin{array}{l}\text { Q-Tag für Arbeit in den } \\
\text { Pädagogischen Teams } \\
\text { reservieren. }\end{array}$ & $\begin{array}{l}\text { Am ,freien' Nachmittag } \\
\text { Anwesenheitspflicht für } \\
\text { gemeinsames Vorbereiten } \\
\text { festlegen. }\end{array}$ \\
\hline $\begin{array}{l}\text { 5) Wie groß ist unsere } \\
\text { Hoffnung, dass sich } \\
\text { etwas ändert? }\end{array}$ & $\begin{array}{l}8 \\
(1=\text { gering bis } 10=\text { gro } ß)\end{array}$ & $\begin{array}{l}10 \\
(1=\text { gering bis } 10=\text { gro } \beta)\end{array}$ \\
\hline
\end{tabular}

\section{Zielvereinbarung 3: Reduktion der Resignationstendenz}

In zwei Teams ist die Anforderung, die eigenen Ressourcen zu schützen und die eigene Resignationstendenz zu senken, in den Fokus der Teamentwicklungsprozesse gerückt (vgl. Tabelle 5). Aus der Einschätzung der Zielbilanzierung aus der Sicht der Gruppe ist ein Vorankommen ersichtlich. Auch in den metrischen Daten der fragebogenbasierten Einschätzungen zeigt sich in der Interventionsgruppe ein statistisch bedeutsames Absinken der Resignationstendenz (vgl. Tabelle 6). 
Tabelle 5: Reflexion der Leitfragen bei Zielvereinbarung und Zwischeneinschätzung der Fokussierung auf Wesentliches und der Reduktion der Resignationstendenz

\begin{tabular}{|c|c|c|}
\hline Leitfrage: & Zielvereinbarung & Zwischeneinschätzung \\
\hline 1) Wo stehen wir jetzt? & $\begin{array}{l}\text { 4 } \\
\text { (1= am Anfang, 10= am Ziel) }\end{array}$ & $\begin{array}{l}6 \\
\text { (1= am Anfang, 10= am Ziel) }\end{array}$ \\
\hline $\begin{array}{l}\text { 2a) Woran erkennen wir } \\
\text { das? } \\
\text { Was ist bereits } \\
\text { vorhanden? }\end{array}$ & $\begin{array}{l}\text { Viele haben den Eindruck, } \\
\text { es kämen immer mehr } \\
\text { Aufgaben dazu, die zu } \\
\text { steigenden Belastungen } \\
\text { führen und zu einer } \\
\text { resignierten Stimmung } \\
\text { führen. }\end{array}$ & $\begin{array}{l}\text { Wir unterscheiden zwischen } \\
\text { „Musts' und ,Supplements'. }\end{array}$ \\
\hline $\begin{array}{l}\text { 2b) Was hat sich } \\
\text { verändert? }\end{array}$ & Zwischenevaluationsfrage & $\begin{array}{l}\text { Wir wägen gemeinsam ab, } \\
\text { ob wir eine zusätzliche } \\
\text { Aufgabe annehmen wollen } \\
\text { oder nicht. }\end{array}$ \\
\hline $\begin{array}{l}\text { 2c) Was haben wir aktiv } \\
\text { beigetragen, damit sich } \\
\text { etwas verändern } \\
\text { konnte? }\end{array}$ & Zwischenevaluationsfrage & $\begin{array}{l}\text { Sich steuern, weniger } \\
\text { klagen, Freude gezielt } \\
\text { erkennen und zeigen. }\end{array}$ \\
\hline $\begin{array}{l}\text { 3) Was können wir aktiv } \\
\text { beitragen, um weiter zu } \\
\text { kommen? }\end{array}$ & $\begin{array}{l}\text { Aufgaben gezielt prüfen, } \\
\text { Schwerpunkte setzen: } \\
\text { Mögliche Aktivitäten und } \\
\text { Aufgaben sammeln, } \\
\text { zwischen ,Must' und } \\
\text {,Supplement' unterscheiden. }\end{array}$ & $\begin{array}{l}\text {,Musts' akzeptieren und } \\
\text { keine Energie für } \\
\text { Diskussionen aufwenden, } \\
\text {,Supplements' als } \\
\text { Möglichkeiten der } \\
\text { Bereicherung betrachten. }\end{array}$ \\
\hline $\begin{array}{l}\text { 4) Was kann die } \\
\text { Schulleitung dazu } \\
\text { beitragen? }\end{array}$ & $\begin{array}{l}\text { Şchulprogramm prüfen, vor } \\
\text { Überfrachtung bewahren }\end{array}$ & $\begin{array}{l}\text { Mit Fragen: „Wollen wir das } \\
\text { wirklich?" Grenzen } \\
\text { markieren. }\end{array}$ \\
\hline $\begin{array}{l}\text { 5) Wie groß ist unsere } \\
\text { Hoffnung, dass sich } \\
\text { etwas ändert? }\end{array}$ & $\begin{array}{l}6 \\
(1=\text { gering bis } 10=\text { groß })\end{array}$ & $\begin{array}{l}7 \\
(1=\text { gering bis } 10=\text { groß })\end{array}$ \\
\hline
\end{tabular}

Ergebnisse der statistischen Prüfung der zielvereinbarungsinduzierten Entwicklungen

Die varianzanalytische Prüfung der Mittelwertsunterschiede zwischen dem ersten und dem zweiten Erhebungszeitpunkt zeigen in allen drei Zielbereichen Veränderungen, die durch die Zielsetzungen induziert wurden. In den Interventionsgruppen lassen sich statistisch signifikante Entwicklungen identifizieren, in den Kontrollgruppen sind keine bedeutsamen Veränderungen erkennbar.

Tab. 6: Vergleich von Entwicklungen zwischen Interventions- und Kontrollgruppe bezüglich von Merkmalen kollektiver Ressourcen, auf welche sich die Zielvereinbarungen beziehen (Fragebogenerhebungen t1 und t2)

\begin{tabular}{|c|c|c|c|c|c|c|}
\hline Gruppe & $\mathrm{n}$ & $\mathrm{M}(\mathrm{SD}) \mathrm{t} 1$ & $\mathrm{M}(\mathrm{SD}) \mathrm{t} 2$ & $p$ & Etasq & Cohen's d \\
\hline \multicolumn{7}{|c|}{ Zielvereinbarung 1: Einheitliche Handhabung der Aufsicht in den Pausen } \\
\hline Interventionsgruppe & 43 & $3.93(1.16)$ & $4.42(.70)$ & $.01^{* *}$ & .149 & .60 \\
\hline Kontrollgruppe & 20 & $4.36(1.34)$ & $4.07(1.44)$ & .54 (n.s.) & .03 & -.31 \\
\hline \multicolumn{7}{|c|}{ Zielvereinbarung 2: Unterrichtsbezogene Kooperation stärken } \\
\hline Interventionsgruppe & 27 & $3.75(.92)$ & $4.26(.92)$ & $.005^{\star *}$ & .309 & .79 \\
\hline Kontrollgruppe & 36 & $3.65(.79)$ & $3.83(.92)$ & .26 (n.s.) & .036 & .33 \\
\hline \multicolumn{7}{|c|}{ Zielvereinbarung 3: Reduktion der Resignationstendenz } \\
\hline Interventionsgruppe & 26 & $2.62(.59)$ & $2.34(.81)$ & $.018^{*}$ & .203 & -.67 \\
\hline Kontrollgruppe & 37 & $2.89(.90)$ & $2.72(.99)$ & .23 (n.s.) & .038 & -.28 \\
\hline
\end{tabular}




\section{Abschluss und Diskussion}

Aus den dargelegten Ergebnissen geht hervor, dass individuelle Ressourcen, wie unterschiedliche Aspekte der Selbstwirksamkeit, für die Einschätzung von Qualitätsmerkmalen des Kollegiums von Bedeutung sind. Damit konnten bisherige Befunde aus dem Schulentwicklungsprojekt RUMBA (vgl. Keller-Schneider/Albisser 2011 und 2012a) durch weitere Facetten angereichert werden. Zudem konnte gezeigt werden, dass bei Einigung über zu verfolgende Ziele eine Entwicklung in die angestrebte Richtung erreicht werden kann, trotz unterschiedlicher Sichtweisen zu Beginn der Intervention. Dieser Befund bestärkt die Bedeutsamkeit von gemeinsam getragenen Zielvereinbarungen.

Dass trotz unterschiedlicher Ausgangslagen, d.h. trotz fehlender Einigkeit darüber, wie eine Situation ,ist', bzw. wie die Qualität eingeschätzt wird, Veränderungen durch Schulentwicklungsprozesse eingeleitet werden können, wenn der Einigkeit über die zu verfolgenden Ziele Gewicht beigemessen wird. Im Rahmen von Schulentwicklungsprozessen in die Zielfindung zu investieren und diese partizipativ zu entwickeln erscheint damit für das Gelingen von Schulentwicklungsprozessen von großer Bedeutung. Da die Zielvereinbarungen und die Einschätzungen der Ausgangssituation sowie die Bilanz bei Abschluss der Intervention in den Teams erfolgte, ist der direkte Effekt von individuellen Merkmalen nicht nachweisbar.

\section{Literatur}

Altrichter, H./Maag Merki, K (Hrsg.) (2010): Handbuch Neue Steuerung im Schulsystem. Wiesbaden.

Bauer, K.O./ Kopka, A./ Brindt, S. (1996): Pädagogische Professionalität und Lehrerarbeit. Eine qualitative empirische Studie über professionelles Handeln und Bewusstsein. Weinheim.

Bauer, K.-O. (2008): Lehrerinteraktion und -kooperation. In: W. Helsper \& J. Böhme (Hrsg.), Handbuch der Schulforschung, S. 839-856. Wiesbaden.

Blömeke, S./ Kaiser, G./ Lehmann, R. (Hrsg.) (2008): Professionelle Kompetenz angehender Lehrerinnen und Lehrer. Wissen, Überzeugungen und Lerngelegenheiten deutscher Mathematikstudierender und -referendare. Münster.

Bonsen, M. (2005): Professionelle Lerngemeinschaften in der Schule. In H. G. Holtappels \& K. Höhmann (Hrsg.), Schulentwicklung und Schulwirksamkeit, S. 180-195. Weinheim.

Bonsen, M./ Bos, W.I Rolff, H. G. (2008): Zur Fusion von Schuleffektivitäts- und Schulentwicklungsforschung. In: W. Bos, H. G. Holtappels, H. Pfeiffer, H.-G. Rolff \& R. Schulz-Zander (Hrsg.). Jahrbuch der Schulentwicklung, Bd. 15, S. 11-39. Weinheim \& München.

Bos, W./ Holtappels, H.G./ Pfeiffer, H./ Rolff H.-G./ Schulz-Zander, R. (2008): Jahrbuch der Schulentwicklung Bd. 15.

Bromme, R. (1992): Der Lehrer als Experte. Bern.

Bronfenbrenner, U. (1981): Die Oekologie der menschlichen Entwicklung. Stuttgart.

Bryk, A. S./ Sebring, P. B./ Allensworth, E./ Luppescu, S./ Easton, J.Q. (2010): Organizing Schools for Improvement: Lessons from Chicago: University of Chicago Press.

Buchwald, P./ Hobfoll, St. E. (2004): Burnout aus ressourcentheoretischer Perspektive. In: Psychologie in Erziehung und Unterricht. Jg. 51, S. 247 - 257.

Buchwald, P./ Schwarzer, C./ Hobfoll (Hrsg.) (2004): Stress gemeinsam bewältigen. Göttingen. 
Combe, A./ Gebhard, U. (2009): Irritation und Phantasie. Zur Möglichkeit von Erfahrungen in schulischen Lernprozessen. In: Zeitschrift für Erziehungswissenschaft, Jg. 12 (3), S. 549571.

Combe, A./ Kolbe, U. (2008): Lehrerprofessionalität: Wissen, Können, Handeln. In: W. Helsper, \& J. Böhme (Hrsg.). Handbuch der Schulforschung, S. 857-878. Wiesbaden.

Deci, E.L./ Ryan, R.M. (1993): Die Selbstbestimmungstheorie der Motivation und ihre Bedeutung für die Pädagogik. In: Zeitschrift für Pädagogik 39, S. 223-238.

Dubs, R. (2006): Führung. In: H. Buchen \& H.G. Rolff: Professionswissen Schulleitung, S. 102- 176. Weinheim.

Fend, H. (1998): Qualität im Bildungswesen. Schulforschung zu Systembedingungen, Schulprofilen und Lehrerleistung. Weinheim.

Fend, H. (2008): Schule gestalten: Systemsteuerung, Schulentwicklung und Unterrichtsqualität. Wiesbaden.

Gathen, von der, J. (2006): Die innerschulische Rezeption von Leistungsrückmeldungen aus Large-Scale-Assessments - Grundlagen und Ziele von Fallstudien. In: H. Kuper \& J. Schneewind. Rückmeldung und Rezeption von Forschungsergebnissen, S. 77-88. Münster.

Groeben, N./ Scheele, B. (2010): Das Forschungsprogramm Subjektive Theorien. In: G. Mey \& K. Mruck (Hrsg.). Handbuch Qualitative Forschung in der Psychologie, Teil 1, S. 151165. Wiesbaden.

Groß Ophoff, J./ Koch, U./ Hosenfeld, I./ Helmke, A. (2006): Ergebnisrückmeldung und ihre Perzeption im Projekt VERA. In: H. Kuper \& J. Schneewind (Hrsg.). Rückmeldung und Rezeption von Forschungsergebnissen. Zur Verwendung wissenschaftlichen Wissens im Bildungsbereich, S. 19-40. Münster.

Heckhausen, J./ Schulz, R. (1995): A Life-Span Theory of Control. In: Psychological Review, Jg. 102, H. 2, S. 284-304.

Helsper, W. (2008): Schulkulturen als symbolische Sinnordnungen. In: W. Helsper, S. Busse, M. Hummrich \& R.-T. Kramer (2008). Pädagogische Professionalität in Organisationen, S. 115-148. Wiesbaden.

Hobfoll, S. E. (1989): Conservation of resources. A new attempt at conceptualizing stress. In: American Psychologist, Jg. 44, S. 513-524.

Keller-Schneider, M. (2010): Entwicklungsaufgaben im Berufseinstieg von Lehrpersonen. Beanspruchung durch berufliche Herausforderungen im Zusammenhang mit Kontext- und Persönlichkeitsmerkmalen. Münster.

Keller-Schneider, M. (2013): Schülerbezogene Überzeugungen von Studierenden der Lehrerbildung und deren Veränderung im Rahmen einer Lehrveranstaltung. Lehrerbildung auf dem Prüfstand, Jg. 6 H. 2, S. 178-213.

Keller-Schneider, M./ Albisser, S. (2011): Einschätzungen von Merkmalen der Teamqualität durch die Teammitglieder und ihr Zusammenhang mit individuellen Merkmalen der einzelnen Lehrpersonen. Basel: SGBF, 20.-22. Juni 2011.

Keller-Schneider, M./ Albisser, S. (2012): Einschätzungen der Schulleitungsqualität - eine Frage der individuellen Ressourcen der Einschätzenden? In: Empirische Pädagogik Jg. 26 H. 1, S. 160-179.

Keller-Schneider, M./ Albisser, S. (2013a): Kooperation von Lehrpersonen und die Bedeutung von individuellen und kollektiven Ressourcen. In: M. Keller-Schneider/ S. Albisser \& J. Wissinger (Hrsg.): Professionalität und Kooperation in Schulen. Beiträge zur Diskussion über Schulqualität, S. 33-56. Bad Heilbrunn. 
Keller-Schneider, M./ Albisser, S. (2013b): Formen der Kooperation von Lehrpersonen und ihr Zusammenhang mit Berufsmotiven. In: E. Wannack, S. Bosshart, A. Eichenberger, M. Fuchs, E. Hardegger \& S. Marti (Hrsg.): 4- bis 12-Jährige - ihre schulischen und außerschulischen Lern- und Lebenswelten, S. 319-327. Münster.

Keller-Schneider, M./ Albisser, S. (2014): Ressourcenentwicklung im Umgang mit Berufsanforderungen. RUMBA - ein Forschungs- und Schulentwicklungsprojekt. Bericht zur zweiten Projektphase 2010-2014. Zürich: Pädagogische Hochschule.

Lazarus, R.S./ Launier, R. (1981): Stressbezogene Transaktionen zwischen Person und Umwelt. In: J. R. Nitsch (Hrsg.): Stress, S. 213 - 259. Bern.

LCH (2008): Berufsleitbild und Standesregeln. Dachverband der Schweizer Lehrerinnen und Lehrer. $\quad$ http://www.lch.ch/fileadmin/files/documents/Verlag LCH/LCH-Berufsleitbild Standesregeln.pdf (Zugriff 5. Okt. 2015).

Marzano, R. J. (2003): What works in schools. Translating research into action. Alexandria/VA: ASCD.

Peek, R. (2006): Dateninduzierte Schulentwicklung. In H. Buchen. \& H.-G. Rolff (Hrsg.), Professionswissen Schulleitung, S. 1342-1366. Weinheim \& Basel.

Reusser, K./ Pauli, C./ Elmer, A. (2011): Berufsbezogene Überzeugungen von Lehrerinnen und Lehrern. In: E. Terhart, H. Bennewitz \& M. Rothland (Hrsg.). Handbuch der Forschung zum Lehrerberuf, S. 478-496. Münster.

Rolff, H.-G. (2007): Studien zu einer Theorie der Schulentwicklung. Weinheim.

Schaarschmitt, U./ Fischer, A.W. (1996): AVEM - Arbeitsbezogenes Verhaltens- und Erlebensmuster. Frankfurt/M.

Scheerens, J./ Glas, C./ Thomas, S.M. (2003): Educational Evaluation, Assessment, and Monitoring. A Systematic Approach. Lisse.

Schneewind, J. (2006): Rückmeldungen als Motivator für die Teilnahme an Schulleistungstest? Die Rezeptionsstudie Belesen. In: H. Kuper \& J. Schneewind. Rückmeldung und Rezeption von Forschungsergebnissen. Zur Verwendung wissenschaftlichen Wissens im Bildungsbereich. Münster.

Schweizer, K./ Klieme, E. (2005): Kompetenzstufen der Lehrerkooperation. Ein empirisches Beispiel für das Latent-Growth-Curve-Modell. In: Psychologie in Erziehung und Unterricht Jg. 52, S. 66-79.

Shulman, L. S. (1991): Von einer Sache etwas verstehen: Wissensentwicklung bei Lehrern. In: E. Terhart, (Hrsg.). Unterrichten als Beruf, S. 145-160. Köln und Wien.

Silins, H./ Mulford, B. (2002): Leadership and School Results. In: K. Leitwood, \& Ph. Hallinger (Hrsg.): Second International Handbook of Educational Leadership and Administration, S. 561-612. Dordrecht/ NL.

Stufflebeam, D. L. (1984): CIPP-Model for Programm Evaluation. In: G. F. Madaus, M. Sciven \& D. J. Stufflebeam: Evaluation Model. Viewpoints on Educational and Human Services Evaluation, S. 117-141. Boston.

Schwarzer, R./ Jerusalem, M. (1999): Skala zur Kollektiven Selbstwirksamkeitserwartung. In: R. Schwarzer \& M. Jerusalem (Hrsg.). Skalen zur Erfassung von Lehrer- und Schülermerkmalen. Berlin.

Wenzel, H. (2008): Studien zur Organisations- und Schulkulturentwicklung. In: W. Helsper \& J. Böhme (Hrsg.). Handbuch der Schulforschung, S. 423-447. Wiesbaden.

Wissinger, J. (2013): Schulleitungshandeln und Förderung der Professionalität unter Lehrpersonen. Eine Analyse institutioneller und struktureller Entwicklungsbedingungen. In: M. Keller-Schneider, S. Albisser \& J. Wissinger (Hrsg.). Professionalität und Kooperation in Schulen. Beiträge zur Diskussion über Schulqualität, S. 185-208. Bad Heilbrunn. 


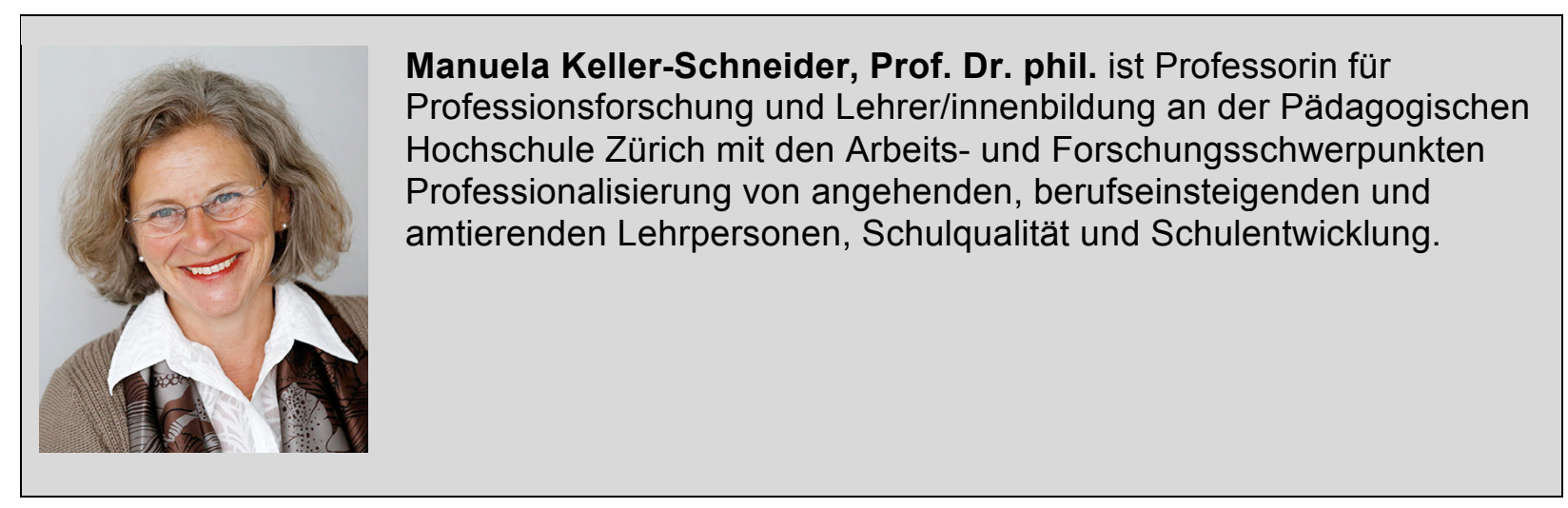

